\title{
Collision Behavior in Magnetic Pressure Parallel Seam Welding of Aluminum Sheets
}

\author{
Akira HATTA $^{1, a}$, Makoto MIYAZAKI ${ }^{1, b^{*}}$ and Yohei KAJIRO ${ }^{1, c}$ \\ ${ }^{1}$ Department of Mechanical Engineering, National Institute of Technology, Nagano College, \\ 716 Tokuma, Nagano-city, Nagano 381-8550, Japan

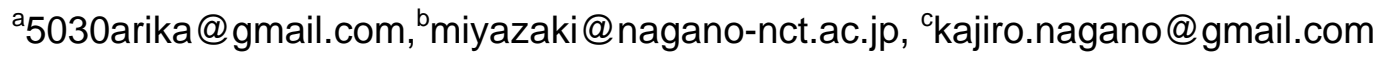

\begin{abstract}
Keywords: Magnetic Pressure Seam Welding, Parallel Seam Welding, Collision Behavior, Aluminum Sheet, Numerical Analysis
\end{abstract}

\begin{abstract}
Magnetic pressure seam welding has attracted attention as a new joining method for aluminum thin plates. Magnetic pressure seam welding is a collision welding process, utilizing electromagnetic force as the acceleration mechanism. The electromagnetic seam welding is a method of abruptly adding a high density magnetic flux around a metal material and utilizing the generated electromagnetic force to deform the thin plate at high speed and pressure welding. This paper deal with the deformation behavior of parallel aluminum seam welded aluminum sheet. Numerical analysis of the dynamic deformation process of the metal plate is performed by the finite element method. The sample used for this analysis is assumed to be a thin plate made of aluminum (A1050-H24, width100mm, thickness $1 \mathrm{~mm}$ ) and composed of quadrilateral elements of plane strain. The experimental results show that the collision speed between the aluminum plates is sufficiently reproduced. The impact point velocity between the aluminum plate surfaces was very high at the initial collision point but decreased continuously during welding. It was also found that the smaller the gap is, the faster the collision point moving speed becomes.
\end{abstract}

\section{Introduction}

Aluminum has higher electrical conductivity and thermal conductivity than iron, so welding is difficult due to low heating efficiency. In previous studies, there is a report on the magnetic pressure seam welding method [1]-[14]. Magnetic pressure seam welding is a collision welding process similar to explosive welding and utilizes electromagnetic force as an acceleration mechanism. Magnetic pressure seam welding accelerates and collides a certain metal plate (flyer plate) to another stationary metal plate (parent plate) by using electromagnetic force. When an impulse current from a capacitor bank passes through a flat one-turn coil, a magnetic flux is instantaneously generated in the coil. The eddy currents are induced in insulated flyer plate in the coil. In magnetic pressure parallel seam welding, one-turn coils are arranged in parallel. A part of flyer plate along the longitudinal direction of the coil bulged toward a parent plate, then flyer plate collided and was welded to a parent plate. At the time of the high-speed collision, metal jets are emitted in the welding interface of the specimen [7]. The collision point velocity and collision angle are determined by the primary and induced electromagnetic force. True metallic bonding is achieved at the mating interface if contact takes place above an appropriate collision point velocity and collision angle [15]. The purpose of this paper is to discuss, the dynamic deformation behavior of magnetic pressure parallel seam welding of aluminum sheets.

\section{Welding principle}

The welding principle is shown in Fig. 1. Magnetic pressure parallel seam welding uses electromagnetic force to accelerate one metal sheet (flyer plate) against another stationary metal 
sheet (parent plate). When a high magnetic field B suddenly occurs and enters the metal sheet, eddy current (current density i) passes through the metal sheet. As a result, the electromagnetic force of Eq. 2 acts mainly on the flyer plate and it is accelerated away from the coil and collides rapidly with the parent plate [10]. The eddy current $\mathbf{i}$, electromagnetic force $\mathbf{f}$ and Joule heat $Q$ are given as follows. $\kappa$ and $\mathbf{B}$ are electric conductivity and magnetic flux density at aluminum sheet. When the residual inductance of the electromagnetic forming apparatus is large, it becomes difficult for a large current to flow through the one-turn coil, so the magnetic pressure also becomes small and it is difficult to join. Since the inductance of the coil of the multi-turn coil is higher than that of the one-turn coil, the current flowing through the coil can be increased and the magnetic pressure can be increased.

$$
\begin{aligned}
& \operatorname{rot} \mathbf{i}=-\kappa \frac{\partial \mathbf{B}}{\partial t} \\
& \mathbf{f}=\mathbf{i} \times \mathbf{B} \\
& Q=\frac{\boldsymbol{i}^{2}}{\boldsymbol{\kappa}}
\end{aligned}
$$

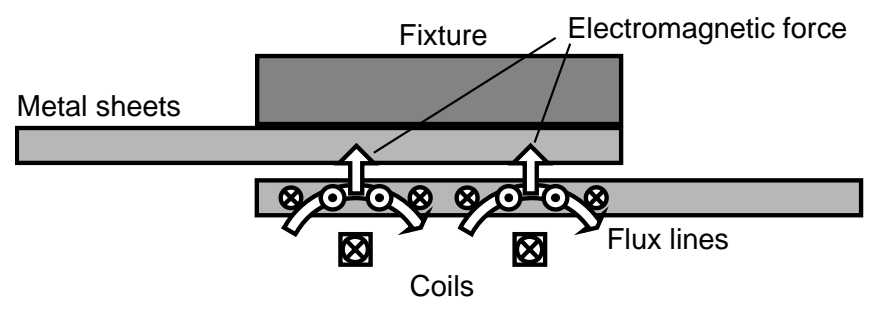

(a) before

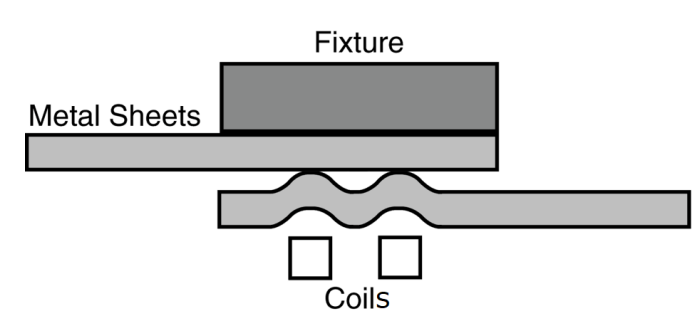

(b) after

Fig. 1 Schematic illustrations of welding method

\section{Numerical Analysis}

The analysis was made by non-linear-structure-analysis-program (MARC 2018). An element division model and boundary condition are shown in Fig. 2. In this analysis, the metal sheets (100mm width, 1mm thickness) were assumed to be composed of 20000 plane-strain quadrilateral elements. The gap length between the flyer plate and the parent plate was varied between from $0.5 \mathrm{~mm}$ to $1.5 \mathrm{~mm}$. The deformed plate was an isotropic material. The true stresstrue strain relation is given by Eq. 4 .

$$
\sigma=F \varepsilon^{n}
$$

The specimens used in this simulation were aluminum sheets. Material properties obtained by a static tension test are shown in Table 1. Okagawa et al. reported that deformation of aluminum sheets was finished at the first discharge waveform [16]. In the analysis, calculation time was $10.8 \mu \mathrm{s}$, and calculation step was 5000. The time integration method was single-step houbolt of implicit solution method. The magnetic pressure $\mathrm{P}$ - measured magnetic flux density $B$ relations are given by Eq. 5 .

$$
P=\frac{B}{2 \mu}\left\{1-\exp \left(-\frac{2 t}{\delta}\right)\right\}
$$




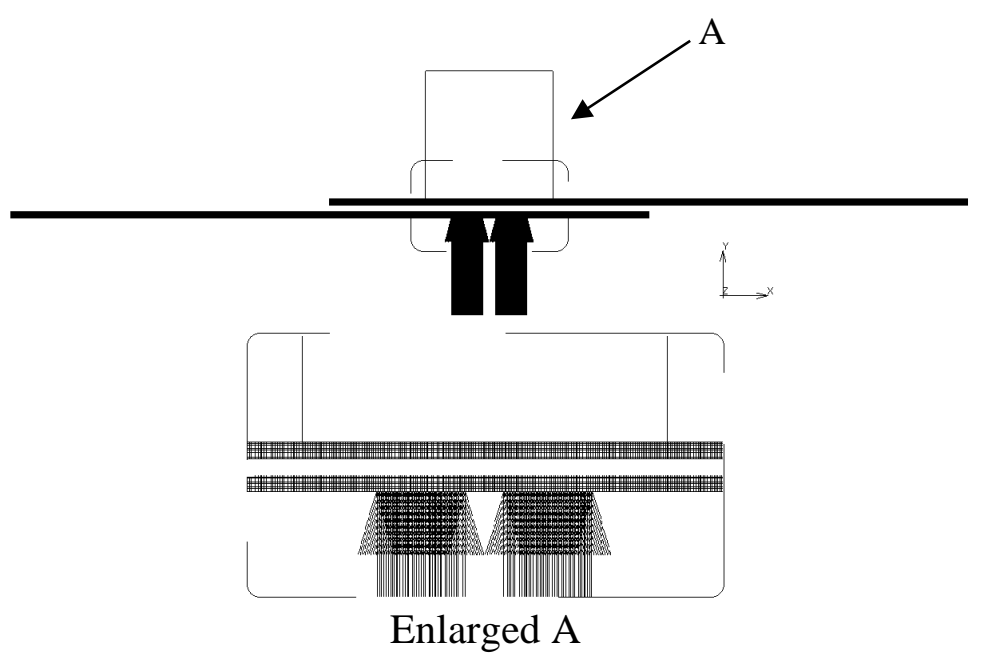

Fig. 2 Finite element model of magnetic pressure paralell seam welding.

Table 1 Material properties of A1050-H24 sheets

\begin{tabular}{cc|c}
\hline Young's modulus & $E[\mathrm{GPa}]$ & 69 \\
\hline Poisson's ratio & $v$ & 0.33 \\
\hline Density & $\rho\left[\mathrm{kg} / \mathrm{m}^{3}\right]$ & $2.71 \times 10^{3}$ \\
\hline Strength coefficient & $F[\mathrm{MPa}]$ & 118 \\
\hline Strain hardening exponent & $\mathrm{n}$ & 0.0623 \\
\hline
\end{tabular}

The parameters $\mu, \delta$ and $t$ are magnetic permeability, skin depth and thickness of metal sheets, respectively. The relationship between time and magnetic pressure is shown in Fig. 3.

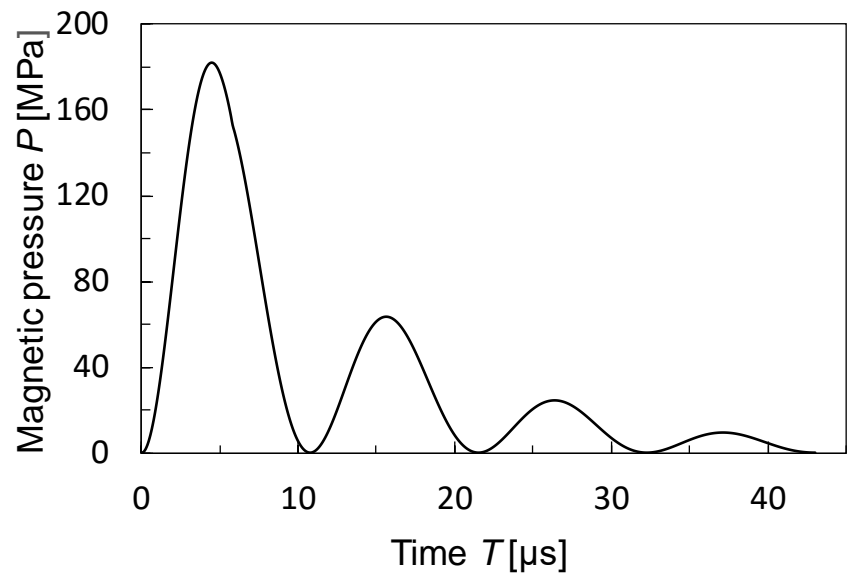

Fig. 3 Relationship between time T and magnetic pressure $P$.

\section{Results and considerations}

The analysis in this study was examined from the initial collision point to the outside. 
Collision angle. The collision angle and collision point velocity relations[15] are given as follows:

$$
\begin{aligned}
& v_{p}=2 v_{c} \sin \frac{\beta}{2} \\
& v_{p}=\sqrt{v_{x}^{2}+v_{y}^{2}}
\end{aligned}
$$

Where $\beta, v_{\mathrm{c}}, v \mathrm{p}, v_{\mathrm{x}}$ and $v_{\mathrm{y}}$ are collision angle, collision point velocity, collision velocity, $\mathrm{x}$ direction element of $v_{\mathrm{p}}$ and y direction element of $v_{\mathrm{p}}$, respectively. Spatial relationships of $v_{\mathrm{p}}, v_{\mathrm{c}}$ and $\beta$ are shown in Fig. 4.

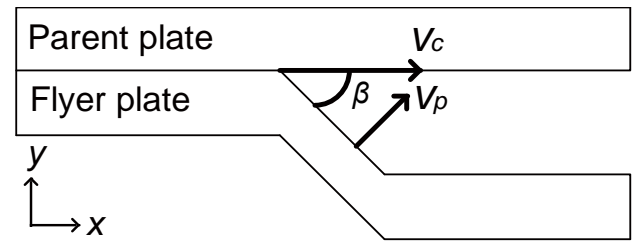

Fig. 4 Definition of collision angle $\beta$, collision velocity $v_{p}$ and welding velocity $v_{c}$.

The relation between distance from initial collision point and collision angle is shown in Fig. 5. Watanabe et al. reported that the collision angle between the metal plate surfaces was 0 degree at the initial collision point [17]. In this simulation, the collision angle between the metal plate surfaces was 0 degree at the initial collision point, but it increased continuously during the welding. Also, increasing the gap length increased the maximum value of the collision angle. When the gap length was $1.5 \mathrm{~mm}$, the collision angle sharply increased at about $2 \mathrm{~mm}$ from the initial collision point.

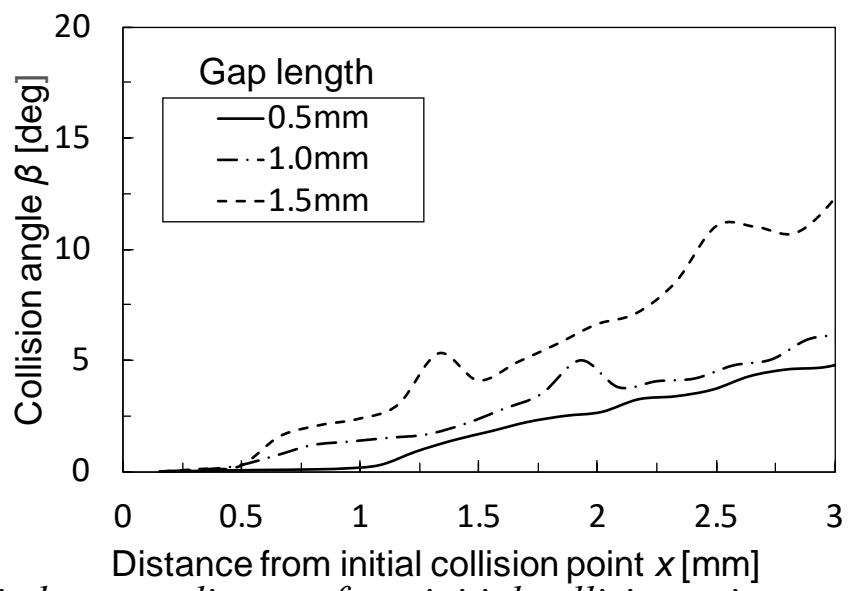

Fig. 5 Relationship between distance from initial collision point $x$ and collision angle $\beta$.

Collision velocity. The relation between initial collision point and collision velocity is shown in Fig. 6. Depending on the gap length, the collision speed is fast. The collision speed became the maximum value at a certain distance away from the initial collision point and decelerated from there. This phenomenon occurs when the movable plate continues to be accelerated by electromagnetic force. The collision speed varies with the electromagnetic force and the mechanical characteristics of the movable plate. 


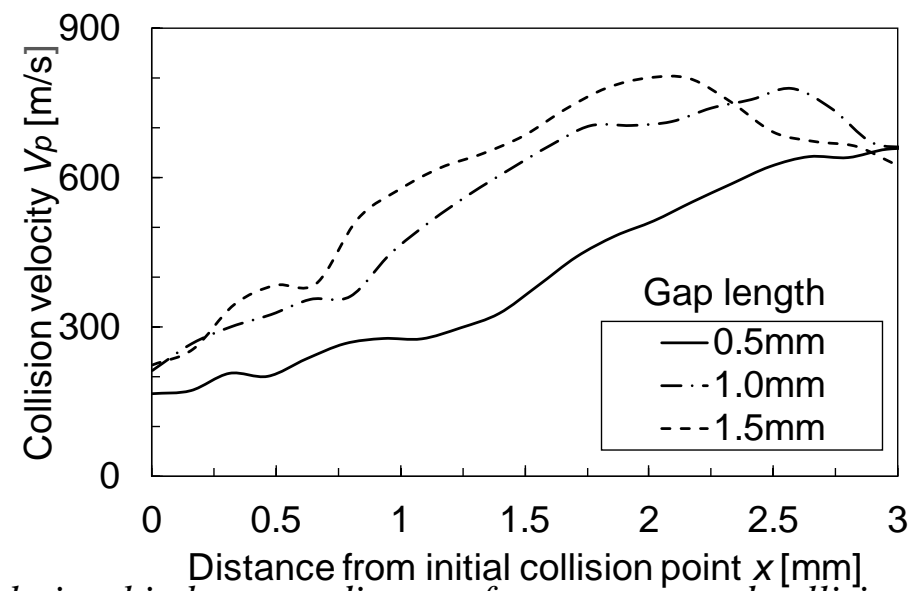

Fig. 6 Relationship between distance from centerxand collision velocity $v_{p}$.

Collision point velocity. The relation between initial collision point and collision point velocity is shown in Fig. 7. The collision point velocity was very high-speed at the initial collision point, but it decreased continuously during the welding. As the gap length increases, the initial collision point movement speed in the vicinity of the initial collision point also increases. When surface layer of the metal plate is emitted as a metal jet, strong metallic bond is established on the metal plate [18]. The velocity of metal jet is $2000-3000 \mathrm{~m} / \mathrm{s}$ [19]. As seen in Fig. 7, the collision point velocity of near the initial collision point is higher than velocity of metal jet. The metal jet is not emitted near the initial collision point. Therefore, the un-welded zone exist near the initial collision point.

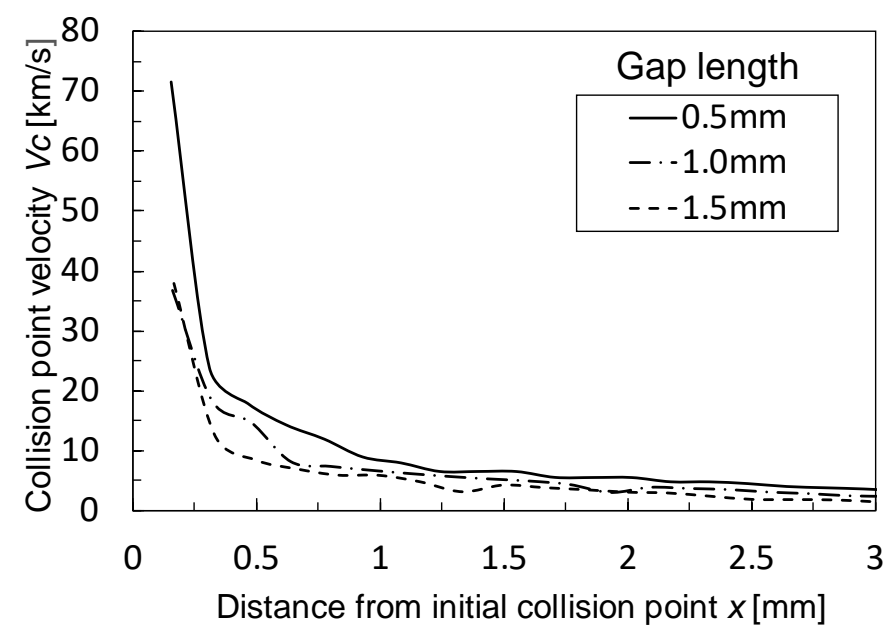

Fig. 7Relationship between distance from initial collision point $x$ and collision point velocity $v_{c}$.

\section{Conclusions}

(1) The collision point velocity is very high-speed at the initial collision point, but it decreases continuously during the welding.

(2) The collision angle between the metal plate surfaces is 0 degree at the initial collision point, but it increases continuously during the welding.

(3) As the gap length increases, the maximum value of the collision angle increases.

(4) The maximum value of the crash velocity tends to increase as the gap length increases.

(5) The analysis from the initial collision point to the outer side is similar to the single coil. 


\section{Acknowledgment}

This work was supported by a Grant-in-Aid for Scientific Research (C) (16K06757) from Japan Society for the Promotion of Science (JSPS).

\section{References}

[1] T. Aizawa, K. Okagawa, M. Yoshizawa and N. Henmi: Proc. of 4th Int. Symp. on Impact Engineering (2001) 827-832.

[2] T. Aizawa: J. of Japan Inst. of Light Metals, 54 (2004) 153-158.

[3] K. Okagawa and T. Aizawa: J. Jpn. Soc. Technol. Plast., 48 (2007) 323-327.

[4] M. Watanabe, S. Kumai and T. Aizawa: Mater. Sci. Forum, 519-521 (2006) 1145-1150.

[5] K. J. Lee, S. Kumai, T. Arai and T. Aizawa: Mater. Sci. Eng. A, 471 (2007) 95-101.

[6] T. Aizawa, M. Kashani and K. Okagawa: Weld. J., 86 (2007) 119s-124s.

[7] M. Watanabe and S. Kumai: Mater. Trans., 50 (2009) 2035-2042.

[8] S.D. Kore, P.P.Date and S.V. Kulkarni: Int. J. of Impact Eng., 34 (2007) 1327-1341.

[9] H. Serizawa, I. Shibahara, S. Rashed and H. Murakawa: Mater. Sci. Forum, 638-642 (2010) 2166-2171.

[10]T. Aizawa, K. Okagawa, M. Kashani: J. Mater. Process. Technol, 213-7 (2013) 1095-1102.

[11]A.Stern, O. Becher, A.Stern, M. Nahmany, D. Ashkenazi, V. Shribman: Weld. J., 94 (2015) 257S-264S.

[12]T. Aizawa, K. Matsuzawa: J. of Jpn. Weld. Soc., 33 (2015) 130s-134s.

[13]M. Watanabe, S. Kumai, K. Okagawa, T. Aizawa: Aluminium Alloys, 2 (2008) 1992-1997.

[14]M. Miyazaki, K. Sasaki and M. Okada: Mater. Sci. Forum, 767 (2014) 166-170.

[15]K. Hokamoto, M. Fujita, M. Ohtsuka: Impact Forming (High-energy Rate Forming), CORONA Publishing Inc., Tokyo, (2017) 66-74.

[16]K. Okagawa and T. Aizawa: J. Jpn. Soc. Technol. Plast., 47 (2006) 632-636.

[17]M. Watanabe, S. Kumai, K. Okagawa, T. Aizawa: Pre-Prints of the 82nd National Meeting of JWS, (2008) 122-123.

[18]M. Watanabe and S. Kumai: Mater. Trans., 50 (2009) 286-292.

[19]S. Kakizaki, M. Watanabe and S. Kumai: Proc. of the 12th Int. Conf. on Aluminium Alloys (2010) 945-949.

[20]K. Okagawa, M. Ishibashi, E. Kabasawa and H. Yamagishi: Proc. of the 66th Jpn. Joint Conf. for the Technol. of Plast., (2015) 389-390.

[21]S. Kakizaki, M. Watanabe and S. Kumai: Mater. Trans., 52 (2011) 1003-1008. 